Service social

\title{
La contribution de l'évaluation à la démocratisation du système de santé et de services sociaux
}

\section{Mireille Tremblay}

Volume 47, numéro 1-2, 1998

Évaluation - Colloque 1999

URI : https://id.erudit.org/iderudit/706782ar

DOI : https://doi.org/10.7202/706782ar

Aller au sommaire du numéro

\section{Éditeur(s)}

École de service social de l'Université Laval

ISSN

1708-1734 (numérique)

Découvrir la revue

Citer cet article

Tremblay, M. (1998). La contribution de l'évaluation à la démocratisation du système de santé et de services sociaux. Service social, 47(1-2), 71-89.

https://doi.org/10.7202/706782ar
Résumé de l'article

Au cours des dernières décennies, l'évaluation des services, des programmes et des politiques a connu une évolution marquée. Alors que sur la scène épistémologique se sont affrontés les modèles positivistes et constructivistes, dans l'arène politique plusieurs catégories d'acteurs ont tenté de dominer les choix publics. Si la maîtrise de savoirs scientifiques, méthodologiques et techniques des uns est indispensable à la compréhension des enjeux en présence, la légitimité des autres est la seule susceptible d'assurer un véritable consensus démocratique. L'évaluation des politiques, des programmes et des services doit contribuer à rationaliser et à améliorer la qualité des services; elle devra aussi créer un espace convivial de négociation des enjeux et des pratiques, un univers de significations et de valeurs où individus et communautés pourront faire des choix légitimes et construire ensemble une société véritablement démocratique. 


\section{La contribution de l'évaluation à la démocratisation du système de santé et de services sociaux}

Au cours des dernières décennies, l'évaluation des services, des programmes et des politiques a connu une évolution marquée. Alors que sur la scène épistémologique se sont affrontés les modèles positivistes et constructivistes, dans l'arène politique plusieurs catégories d'acteurs ont tenté de dominer les choix publics. Si la maîtrise de savoirs scientifiques, méthodologiques et techniques des uns est indispensable à la compréhension des enjeux en présence, la légitimité des autres est la seule susceptible d'assurer un véritable consensus démocratique. L'évaluation des politiques, des programmes et des services doit contribuer à rationaliser et à améliorer la qualité des services; elle devra aussi créer un espace convivial de négociation des enjeux et des pratiques, un univers de significations et de valeurs où individus et communautés pourront faire des choix légitimes et construire ensemble une société véritablement démocratique.

During the last decades, the evaluation of services, programs and policies has evolved considerably. While on the epistemological scene tenants of positive and constructive theoretical models opposed each other, on the political scene many actors tended to dominate public choices. The evaluation of policies, programs and services must not only contribute to the rationalization and to the upgrading of service quality. It must also create a convivial space for the negotiation of stakes and practices, a world of significant meanings that let individuals and communities make legitimate and transparent choices in order to build together a real democratic society. 


\section{INTRODUCTION}

Deux enjeux majeurs sous-tendaient la réforme de la santé et des services sociaux au début de la décennie, soit rationaliser et démocratiser le système de senvices. L'objectif de rationalisation visait l'amélioration de la qualité et de l'efficacité des services; on cherchait à contrôler les dépenses en adaptant les services aux besoins de la population, d'une part, et aux ressources de l'État, d'autre part. L'objectif de démocratisation concernait l'engagement des citoyens et des citoyennes dans un processus de décision collectif et décentralisé aux paliers régional et local.

Ces objectifs ont été clairement énoncés par la Commission d'enquête sur les services de santé et les services sociaux, plus communément appelée commission Rochon, qui proposait les orientations suivantes comme fondements de sa réforme afin de revitaliser le système de santé et de services sociaux :

- un système axé sur des résultats à atteindre;

- une démocratisation et une plus grande transparence des processus décisionnels, en particulier par une participation accrue des citoyens aux diverses instances du système;

- une décentralisation et une meilleure adaptation aux réalités régionales;

- une approche intersectorielle des problèmes de santé et de bienêtre, tant au niveau local qu'aux niveaux régional et provincial;

- une approche de population qui permette d'agir efficacement sur les facteurs déterminants des problèmes de santé et des problèmes sociaux;

- un système public sur les plans du financement et du fonctionnement (Commission d'enquête sur les services sociaux et les services de santé, 1987).

Ces orientations formulées il y a maintenant une dizaine d'années semblent toujours d'actualité. Alors que la question de la rationalisation des dépenses a dominé la scène publique depuis le début des années 1990 et a culminé plus particulièrement dans le cadre des compressions budgétaires, à partir de la deuxième moitié de cette même décennie peu d'efforts ont visé l'accroissement des pouvoirs 
des citoyens et des citoyennes dans une réforme que le ministre Côté souhaitait axée sur le " le citoyen décideur, consommateur et payeur ".

Nous montrerons dans les pages suivantes pourquoi et comment l'évaluation des politiques, des programmes et des services doit contribuer à atteindre l'objectif de démocratisation du système de santé et de services sociaux. Après avoir rappelé la complexité et les dimensions de l'évaluation, nous verrons comment cette pratique a évolué au Québec dans le réseau de la santé et des services sociaux. Nous étudierons plus particulièrement l'évolution croissante et l'importance de l'implication des acteurs et des actrices légitimement concernés par un débat que nous souhaitons véritablement démocratique.

\section{LES DIMENSIONS DE L'ÉVALUATION : MESURER OU DÉLIBÉRER?}

Depuis la mise en place du réseau sociosanitaire québécois, plus d'un a insisté sur l'importance de l'évaluation, qui a connu une évolution constante. On reconnaît maintenant que l'évaluation recouvre une réalité complexe comportant plusieurs dimensions. Louis Demers la définissait ainsi, dans un document rédigé à la demande de la commission Rochon et portant sur le bilan de l'évaluation dans le réseau de la santé et des services sociaux :

La problématique de l'évaluation dans le domaine de la santé est fort complexe. Dans sa plus grande extension, son objet va de la qualité des actes professionnels à la pertinence des politiques ministérielles en passant par les programmes et les stratégies d'intervention. Sa définition peut englober aussi bien les jugements personnels des usagers, les données statistiques de la RAMQ que les recherches évaluatives à caractère scientifique (Demers, 1987, p. 1).

Cette conception de l'évaluation repose sur deux dimensions, soit l'objet de l'évaluation et la méthode utilisée. Sur le plan de l'objet de l'évaluation, la définition proposée inclut aussi bien les évaluations macroscopiques des politiques ministérielles ou des programmes régionaux que les évaluations plus restreintes des services et des interventions professionnelles dont les effets sont parfois mesurables à une échelle microscopique. Sur le plan méthodologique, la définition de Louis Demers témoigne d'une grande diversité quant aux sources et au mode de traitement des données, l'évaluation pouvant faire usage d'approches subjectives ou de méthodes rigoureuses, objectives et scientifiques. 
Malgré la diversité des objets de l'évaluation et des méthodes utilisées, le but de l'évaluation fait l'unanimité parmi les professionnels : il s'agit toujours de porter un jugement sur une politique, un programme ou un service, pour en augmenter la rationalité (l'efficacité, l'efficience) ou pour en accroître l'acceptabilité sociale et politique (la pertinence). L'évaluation permet de faire des choix non seulement sur la manière de faire les choses, mais également sur la nécessité d'intervenir. On parlera d'évaluation formative quand on cherche à améliorer la qualité d'une intervention, et d'évaluation sommative lorsqu'on documente son utilité et qu'on doit décider du maintien ou de l'abolition d'un programme.

L'évaluation s'inscrit dans un processus de prise de décision. Elle constitue l'une des étapes indispensables du cycle de planification des politiques, des programmes et des services publics. La première étape de ce cycle consiste à circonscrire les problèmes et les besoins d'une population, la seconde consiste à analyser et à concevoir l'intervention. Pendant la troisième il s'agit de procéder à l'implantation de la politique, du programme ou du service, alors qu'au cours de la dernière étape on procède à l'évaluation des résultats obtenus par rapport aux objectifs fixés. Cette dernière étape, soit celle de l'évaluation, permet de porter un jugement sur le programme et de le réviser; c'est ainsi que le cycle recommence (Blum, dans Pineault et Daveluy, p. 61).

En résumé, toute démarche pratique ou de recherche qui tente de recueillir et d'interpréter des informations concernant une politique, un programme, un service ou un acte professionnel à l'intérieur d'un système (gouvernemental, institutionnel, professionnel) afin de porter un jugement sur les interventions effectuées peut être considéré comme un évaluation. Cette démarche vise à porter un jugement et à prendre des décisions sur les politiques, les programmes et les services publics. Quelles que soient la qualité et la validité des informations recueillies et traitées, il s'agira toujours de porter un jugement et c'est tout particulièrement cette finalité qui amène à se pencher sur la légitimité du processus de décision.

Si l'ensemble de la démarche doit contribuer à la rationalisation des services, le processus de décision quant à lui doit assurer la participation légitime des acteurs concernés. Outre les dimensions liées à son objet, à la méthode ou au but visé, l'évaluation comporte des dimensions éthiques et politiques associées à la démocratisation des services. 
Ces dimensions ne peuvent être dissociées des dimensions épistémologiques visant à soutenir la rationalité des décisions qui doivent être prises, et c'est ce que soutenait la Commission d'enquête sur les services de santé et les services sociaux:

Les dimensions éthiques de tels choix ne peuvent être ignorées. La première tâche de ceux et celles qui auront à décider si un services est couvert ou ne l'est pas est d'obtenir un consensus sur les lignes directrices à suivre. Celles-ci devraient tenir compte de l'évolution des problèmes, des valeurs morales de la société et des capacités d'intervention. Dans cette perspective, la préoccupation constante des décideurs devrait être de s'assurer que les services couverts sont vraiment ceux dont la société retire le plus de bénéfice (Commission d'enquête sur les services de santé et les services sociaux, 1987, p. 27).

Si nous pouvons considérer l'évaluation sous plusieurs angles, soit sous l'angle de l'objet, de la méthode ou des buts recherchés, le rôle des acteurs doit susciter une attention particulière. Qui seront les utilisateurs de l'évaluation, qui sont les participants et participantes qui transmettent des informations ou portent des jugements, qui sont les personnes qui réalisent l'évaluation? La réponse à ces questions met en lumière des intérêts parfois divergents qui doivent être clarifiés si l'on veut garantir la légitimité d'une démarche d'évaluation. C'est en tenant compte de la dimension des acteurs que s'est effectuée la réforme d'inspiration " constructiviste " dans la pratique évaluative au Québec. On considère généralement l'approche constructiviste comme une "quatrième génération en évaluation de programmes ".

\section{ÉMERGENCE ET STRUCTURATION DE L'ÉVALUATION ET DE LA RECHERCHE ÉVALUATIVE}

La croissance et la multiplication des pratiques d'évaluation des programmes publics ont suivi l'évolution et la croissance de l'intervention de l'État au cours du XXe siècle. L'évaluation s'est développée parallèlement aux pratiques de planification publique qui ont connu une expansion fulgurante avec la montée de l'État-providence. Avec la remise en question du rôle de l'État, la popularité de l'évaluation n'a pas fléchi. Au contraire, elle s'est accrue d'autant que l'on comptait sur l'évaluation pour contribuer à la rationalisation des dépenses publiques. 
Au Québec, le Conseil du trésor a fait la promotion de l'évaluation des programmes dès 1970, par un ensemble d'activités visant essentiellement la rationalisation des services publics, comme :

- la mise à l'essai de la technique des mémoires de programmes, au milieu des années 1970,

- la mise sur pied d'équipes d'évaluation de programmes au Secrétariat du Conseil du trésor, à la fin de la même décennie,

- la mise en place d'équipes spécialisées dans quelques ministères et organismes, surtout depuis 1989,

- un projet de politique cadre, approuvé en principe par le Conseil du trésor en 1984 et qui confiait aux ministères et aux organismes le soin de bâtir leur propre plan d'évaluation,

- la formation d'un groupe de travail interministériel, chargé d'approfondir les liens entre l'évaluation de programme et le processus de décision et d'allocation des ressources, et dont un premier rapport a été déposé en janvier 1986 (Garneau, Poitras, Savard, 1986, p. 3).

Dans le réseau de la santé et des services sociaux québécois, on peut considérer que l'évaluation et la recherche évaluative - qui répond à des critères plus rigoureux sur le plan de la méthodologie n'ont connu de véritable essor que dans le courant des années 1980, s'accélérant dans le courant des années 1990.

En 1976, le ministère de la Santé et des Services sociaux mettait en place deux services d'évaluation, l'un pour le secteur de la santé et le second pour le secteur social. Ces deux services étaient fusionnés en 1982 en une direction de l'évaluation des programmes, et en 1985 cette direction devenait la Direction générale de planification et d'évaluation (D’Annunzio, 1986).

À cette époque le modèle théorique de référence, publié en 1985 par Pierre Bergeron, alors directeur, était de type systémique, inspiré par les travaux du Groupe interdisciplinaire de recherche de l'Université de Montréal (GRIS). Si la recherche évaluative au ministère de la Santé et des Services sociaux était concentrée dans cette direction générale, d'autres directions ont assumé des tâches liées à l'évaluation : analyses financières, suivi et "monitoring " des programmes, mise 
sur pied de systèmes d'information sont autant d'activités menées par les autres directions ministérielles, qui ont contribué à l'essor des pratiques évaluatives pendant les années 1980 et plus particulièrement au cours de la décennie 1990.

Malgré les efforts du Conseil du trésor et du ministère de la Santé et des Services sociaux, en 1982 Gauthier brossait un portrait relativement sombre de la recherche évaluative dans le domaine de la santé au Québec. La qualité globale des recherches laissait trop souvent à désirer selon lui pour l'une ou l'autre des raisons suivantes : plusieurs recherches étaient mal planifiées, les objectifs des programmes étaient imprécis - lorsque ces objectifs avaient été formulés -, les concepts étaient peu opérationnels, les stratégies de vérification et de contrôle des variables étaient absentes ou inadéquates. Les milieux universitaires étaient les premiers producteurs de recherche évaluative avec $36 \%$ des recherches recensées; venaient ensuite les départements de santé communautaire avec $21 \%$ des recherches. Les centres de services sociaux en produisaient $12 \%$ et le ministère de la Santé et des Services sociaux produisait $11 \%$ de ces travaux. Gauthier constatait que les recherches provenant des milieux universitaires et subventionnées par le Conseil québécois de la recherche sociale (CQRS) étaient de qualité supérieure à celles produites par les départements de santé et les autres organismes publics, ces derniers ne disposant que rarement de la concentration de ressources et de l'expertise requise pour mener à terme des recherches évaluatives de haut calibre. Sur le plan de la méthode il observait que $70,7 \%$ des recherches adoptaient des approches quantitatives, alors que $12,1 \%$ optaient pour une démarche qualitative et $17,2 \%$ pour une approche mixte.

En $1989 \mathrm{M}^{\text {me }}$ Lavoie-Roux déplorait toujours la faiblesse de la recherche évaluative au Québec :

La recherche évaluative permet d'apprécier l'efficacité des interventions et des pratiques thérapeutiques et de mesurer l'impact des politiques et des programmes. Malgré son importance, elle souffre mal la comparaison avec certains autres secteurs et un nombre insuffisant de chercheurs s'y consacrent [...] Par l'entremise de ses deux principaux organismes subventionnaires, le Fonds de la recherche en santé du Québec et le Conseil québécois de la recherche sociale, le Ministère envisage de favoriser la mise en œuvre de programmes de subvention destinés à des projets qui 
visent essentiellement l'évaluation des interventions, des programmes et des politiques ainsi que le développement de la recherche organisationnelle (Ministère de la Santé et des Services sociaux, 1989, p. 131).

Une enquête menée par le Fonds de la recherche en santé du Québec (FRSQ, 1990) à la même époque, auprès d'un millier de chercheurs du domaine de la santé, révélait que parmi eux $18 \%$ seulement faisaient de l'évaluation. Ces $18 \%$ de chercheurs sont répartis comme suit : $13 \%$ en recherche évaluative, $2 \%$ en recherche organisationnelle et $3 \%$ en recherche opérationnelle.

Cette situation n'est pas propre au Québec. L'Organisation mondiale de la santé, qui incitait dès 1981 les États membres à évaluer leurs programmes de santé, déplorait en 1987, dans son Septième rapport sur la situation sanitaire dans le monde: Santé pour tous d'ici l'an 2000 , l'absence de projets d'évaluation de la qualité des services de santé.

Cette situation va changer et la volonté politique sera telle, à partir des années 1990, que dans le cadre de la réforme de la santé et des services sociaux de nouveaux acteurs vont s'engager. Au ministère de la Santé et des Services sociaux et aux milieux de recherche parrainée par le FRSQ, le CQRS ou encore les départements de santé communautaire vont se joindre les régies régionales et les établissements. D'autres institutions se sont engagées sur cette voie, comme les associations d'établissements, le Conseil québécois d'agrément, l'Institut de la santé publique.

\section{L'ESSOR DE L'ÉVALUATION POUR RATIONALISER ET DÉMOCRATISER LE SYSTÈME}

La commission Rochon, de même que les divers textes législatifs ou ministériels qui ont émaillé la réforme sociosanitaire au cours des années 1990, a affirmé la nécessité de développer l'évaluation des politiques, des programmes et des services afin de poursuivre les objectifs de rationalisation et de démocratisation du système sociosanitaire et ainsi contribuer à l'amélioration du processus de décision.

La Commission d'enquête sur les services de santé et les services sociaux accordait une place importante à l'évaluation dans la poursuite 
des objectifs de rationalisation et de démocratisation du système de services, objectifs qu'elle estimait complémentaires :

Le financement des services de santé et des services sociaux résultera toujours d'un compromis politique entre la volonté d'atteindre certains objectifs de santé et de bien-être et le coût de réalisation de ces objectifs. Pour qu'une société puisse effectuer les arbitrages nécessaires, il faut que la population soit en mesure d'évaluer les performances du système, de se prononcer sur les objectifs et les priorités et de décider du niveau de financement qui devrait être consacré au secteur de la santé et du bien-être. Elle doit donc disposer de l'information nécessaire et du pouvoir de décision (Commission d'enquête sur les services de santé et les services sociaux, 1987, p. 26).

Tous les textes instituant la réforme du réseau sociosanitaire, faisant suite aux travaux de la commission Rochon, ont également insisté sur la nécessité de développer l'évaluation et la recherche évaluative qui devaient contribuer à " recentrer les services sur les besoins des personnes et sur les objectifs de santé et de bien-être ". Madame Lavoie-Roux dans ses Orientations ministérielles (1989) déplorait le manque d'informations concernant les programmes et les services; elle recommandait de développer la recherche évaluative "qui permet d'apprécier l'efficacité des interventions et des pratiques thérapeutiques et de mesurer l'impact des politiques ou des programmes » (p. 131).

Dans sa « réforme axée sur le citoyen » Marc-Yvan Côté (1991) voulait « recentrer le réseau sur des objectifs de résultats » et, pour ce faire, il mettait à contribution la recherche évaluative. II désirait modifier la dynamique selon laquelle les corporations et les groupes sociaux réclamaient sans cesse de plus en plus de ressources. II promettait alors une politique sociosanitaire québécoise qui énoncerait clairement les résultats attendus, le rôle de l'évaluation consistant ensuite à mesurer l'atteinte de ces objectifs.

En 1991, la Loi sur les services de santé et les services sociaux (L.R.Q., chap. 42) précisait les responsabilités provinciales, régionales et locales en matière d'évaluation des politiques, des programmes et des services. La Loi confiait aux régies régionales la responsabilité d'évaluer, "selon la périodicité que détermine le ministre, l'efficacité des services de santé et des services sociaux, le degré d'atteinte des 
objectifs poursuivis et le degré de satisfaction des usagers à l'égard des services ". Les régies devaient également élaborer et mettre en œuvre, " conformément aux directives du ministre, un programme d'évaluation de la qualité des services offerts par les établissements » (art. $266,5^{\circ}$ et $6^{\circ}$ ).

Au sein des nouvelles régies régionales étaient également instituées des directions de santé publique qui recevaient le mandat d'informer la population « de l'état de santé général des individus qui la composent, des problèmes de santé prioritaires, des groupes les plus vulnérables, des principaux facteurs de risque et des interventions qu'il juge les plus efficaces, d'en suivre l'évolution et, le cas échéant, de conduire des études ou recherches nécessaires à cette fin » (art. 371, $1^{\circ}$ ). L'ampleur de ce mandat a suscité l'accroissement et l'accélération du développement de la recherche évaluative au sein des régies régionales.

Quant aux conseils d'administration des établissements, le législateur leur demandait de s'assurer « de la pertinence, de la qualité et de l'efficacité des services dispensés; du respect des droits des usagers [...] de l'utilisation économique et efficiente des ressources humaines, matérielles et financières " (art. $172,1^{\circ}, 2^{\circ}, 3^{\circ}$ ). La mise sur pied du Conseil québécois d'agrément a soutenu les efforts de plusieurs établissements dans la gestion de la qualité de leurs services. De plus, la plupart des associations d'établissements ont instauré des mécanismes d'évaluation et de suivi des services, des programmes et des politiques pour soutenir leurs membres et défendre leurs intérêts.

Ainsi qu'il avait été prévu, la Politique de la santé et du bien-être, parue en 1992, a précisé 19 objectifs de santé et de bien-être ainsi que six stratégies prioritaires d'intervention. La politique a également réaffirmé la nécessité de développer l'évaluation et la recherche évaluative afin d'améliorer la pertinence, l'efficacité et l'efficience des politiques, des programmes et des services. La publication de ce document et les orientations qu'il contenait ont suscité une vaste entreprise de hiérarchisation des programmes par toutes les régies régionales au Québec et ont contribué à l'essor de divers outils et pratiques d'évaluation : indicateurs de santé et de bien-être, cadres d'évaluation des programmes, indicateurs de besoins ont jailli de toutes parts afin de permettre l'implantation et le suivi de la politique, des objectifs 
et des programmes, tant par le ministère de la Santé et des Services sociaux que par les régies régionales.

Malgré les réserves formulées concernant l'impact réel des pratiques évaluatives au cours de la dernière décennie, comme le soulignaient plusieurs conférenciers (Mercier, Bélanger, Sormany) à l'occasion d'un colloque du Conseil québécois de la recherche sociale tenu en mai 1995, la recherche évaluative a connu un essor réel au cours de la dernière décennie et a vu croître l'intérêt de plusieurs institutions publiques à l'égard de cette pratique aux niveaux provincial, régional et local.

\section{LA RECONNAISSANCE DE L'ACTEUR}

Sur le plan épistémologique, au cours des années 1980 les sciences sociales ont commencé à remettre en question le paradigme empirique en évaluation de politiques et de programmes. L'incapacité des méthodes quantitatives à considérer les aspects subjectifs, leur insensibilité aux valeurs sociales, leur échec à produire des résultats valides en évaluation de programmes, le refus des décideurs de tenir compte des résultats obtenus et des recommandations formulées ont nourri le désenchantement des évaluateurs qui commencent alors à explorer de nouvelles méthodes de recherche en évaluation.

Au cours des années 1990, aux méthodes quantitatives, rationalistes et objectives se sont ajoutées les approches qualitatives, qui accordent une place plus importante aux différents acteurs concernés par une politique, un programme ou un service. Des auteurs comme Patton (1987), Deslauriers (1987) Laflamme (1987) et Nadeau (1988) ont contribué à populariser ce type de recherche au Québec. On parle maintenant d'approche constructiviste et de la " quatrième génération de recherche évaluative " (Guba et Lincoln, 1989).

Quelle que soit la technique retenue, récit de vie, étude de cas, recherche-action, groupe nominal, observation participante, les approches de type qualitatif ont pour avantage de resituer la personne au cœur de l'univers de significations qu'elle habite. La connaissance ne peut plus être considérée comme neutre et objective, elle est socialement et humainement construite. Partant du rôle d'objets d'étude auquel ils sont confinés dans une démarche positiviste et empirique, 
les acteurs sociaux acquièrent le statut de sujets qui donnent une signification aux réalités collectives et sociales qu'ils contribuent à produire.

L'évaluation devient ainsi un lieu et un moteur du changement. La recherche évaluative, en adoptant le paradigme constructiviste, permet d'engager et de manifester des solidarités sociales afin d'énoncer et de produire une société dans toute sa complexité et sa signification (Soulet, dans Deslauriers, 1989). Dans une perspective dite constructiviste, l'évaluation contribue à créer et à construire la réalité sociale. La recherche évaluative n'invoque plus une vérité absolue et définitive, mais elle permet aux différents protagonistes de négocier les enjeux, les objectifs et la valeur d'un programme ou d'un service. La réalité sociale est alors considérée comme le fruit d'une négociation collective. Les personnes concernées sont responsables autant du choix des questions que de celui des méthodes, des conclusions et des recommandations de l'évaluation. Le sujet coexiste avec l'objet et cette coexistence est source de créativité. "C'est cette conscience du soi dans l'objet qui devient le moteur du sujet connaissant » (Soulet, dans Deslauriers, p. 21).

Concevoir ainsi l'évaluation non seulement requiert l'amélioration de nos pratiques évaluatives, mais exige un changement de paradigme. Pour plusieurs il s'agit là d'une révolution plus que d'une évolution. L'approche dite constructiviste repose sur un changement de paradigme scientifique, sur un modèle épistémologique qui doit dépasser les approches positivistes et objectives (Guba et Lincoln, 1989). Avec le paradigme constructiviste, on assiste non seulement au déplacement de la recherche évaluative, des moyens vers les objectifs, comme le soulignait Demers (1987), mais au glissement de l'évaluation, des objectifs vers les enjeux sociaux, et à une ouverture sur les aspects politiques. La quatrième génération en évaluation de programmes redonne une place aux actrices et aux acteurs sociaux, à leur capacité de produire, de négocier et de créer le social. Les problèmes sociaux et de santé mènent inexorablement aux problèmes politiques et, si l'on est en quête de justice et d'égalité, on aborde les rivages de la politique et de l'éthique.

En 1995, lors d'un colloque du Conseil québécois de la recherche sociale portant sur l'évaluation du réseau sociosanitaire, plusieurs chercheurs et chercheuses ont insisté sur la nécessité de tenir compte 
de la diversité des acteurs en présence concernés par une politique ou un programme (Godbout, Mercier, Bélanger, Bouchard, Rodriguez et Guay). Qu'il soit question des actrices et des acteurs politiques, administratifs, professionnels, des usagers et usagères, des citoyens et citoyennes ou des groupes communautaires, toutes les personnes concernées doivent être engagées dans un processus d'évaluation. L'évaluation est alors conçue comme un " processus de négociation " qui peut " devenir un outil de renouvellement du système à travers son ouverture aux intérêts des populations et aux valeurs des nouveaux partenaires " (Rodriguez et Guay, dans CQRS, 1995, p. 160). Pour paraphraser Bélanger (CQRS, 1995) qui discutait de l'impact de la commission Rochon, il n'est pas suffisant d'évaluer pour provoquer un changement de politiques ou de programmes : il faut " par la suite un consensus de société et une volonté politique » (p. 118).

\section{MAIS DE QUEL ACTEUR S'AGIT-IL... ET LA DÉMOCRATIE ALORS?}

Plusieurs catégories d'acteurs doivent être engagés dans l'évaluation des politiques, des programmes et des services. Que ce soient ceux qui demandent que soit menée une évaluation, que ce soient les évaluateurs responsables de la conduire ou les usagers et usagères des interventions faisant l'objet de l'évaluation, tous ont des intérêts qui leur sont propres. La littérature abonde sur les oppositions et les divergences d'intérêts entre décideurs et planificateurs, entre technocrates et intervenants, entre distributeurs de services et usagers qui tous sont concernés par l'évaluation des interventions publiques.

Lors d'un colloque sur l'évaluation des programmes publics au Québec, les participants et participantes se sont inquiétés de l'utilité de la recherche évaluative pour les décideurs et les décideuses. Laurent Laplante (1987) déplorait que les résultats des " évaluations faites par des spécialistes sont si brumeux et incompréhensibles qu'ils deviennent inutilisables ".

Dans le cadre d'une conférence, Richard French (1987), alors ministre canadien, dénonçait le « malentendu fondamental » entre les politiciens et les technocrates, entre ceux qui fixent les objectifs et les finalités et ceux qui choisissent les moyens.

Les décideurs politiques sentent très bien ce que peu d'analystes sont disposés à reconnaître : à savoir que les instruments analytiques des sciences sociales, comme je l'ai déjà fait remarquer, sont 
incapables de rattacher les lignes de conduite aux objectifs, autrement dit les moyens aux fins, assez péremptoirement pour que les technocrates mettent au point des politiques et des programmes qui soient plus ou moins manifestement le prolongement des objectifs déterminés par les politiciens ( $p .7)$.

Si les conflits opposant décideurs et technocrates sont connus, ceux qui opposent les technocrates et la population sont également bien documentés. Dans le cadre des travaux de la commission Rochon le bilan de la participation des usagers, des usagères et de la population au processus de prise de décision était assez sombre, l'appareil technocratique ayant des difficultés à s'adapter aux réalités communautaires (Godbout et collab., 1987; Brassard, 1987). Une autre recherche portant sur I 'évaluation du processus de participation aux plans régionaux d'organisation de services en santé mentale révélait un " choc des cultures » entre les technocrates et le réseau communautaire (Lamoureux, 1994).

Godue et Chambaud (1981), Bibeau (1984), Lesemann et Lamoureux (1987) nous avaient déjà mis en garde contre la récupération du dynamisme communautaire, la technocratisation du système et la dépolitisation des problèmes sociaux transformés en problèmes administratifs. Renaud (1984) déplorait que :

En vingt ans le rêve d'une " société de participation » où des groupes multiples de citoyens devaient intervenir pour influencer les décisions du pouvoir se renverse en cauchemar d'une société habilement manipulée pour obtenir des groupes de citoyens un travail qui adhère aux objectifs de la technocratie ( $p .115)$.

Malgré cette déception, la demande de participation s'est maintenue et plusieurs auteurs ont insisté sur la place qui doit être réservée aux usagers et usagères et à la population, qu'il soit question de promotion de leurs droits, du choix et de la hiérarchisation des programmes, de l'évaluation du degré de satisfaction et de la qualité des services sociaux et de santé (Demers, 1987; Von Schoenberg, 1985; Corin et collab., 1987). Parce que le recours à « la consultation et aux commissions constitue un rempart contre le pouvoir des technocrates » (Bibeau, 1984, p. 24), la recherche évaluative doit s'appuyer sur la participation la plus large et sur la négociation transparente et publique d'enjeux collectifs. 
II vaut la peine de demander à l'usager comment il évalue les services, comment les mécanismes actuels qui lui permettent de jouer un rôle pourraient être améliorés, et de faire le postulat que son évaluation est aussi valable que celle des experts et des autres intervenants (Godbout, 1987, p. 7).

Si la littérature en évaluation et en recherche évaluative accorde une place aux décideurs, aux technocrates, aux planificateurs, aux distributeurs, aux groupes communautaires et aux usagers visés par une politique, un programme ou un service, il est moins fréquent de reconnaître la participation de personnes en tant que simples citoyens et citoyennes. Ellenberger (1981) déplore le glissement de sens de la participation publique des citoyens et des citoyennes vers une logique de production et de consommation de services. Les citoyens et les citoyennes sont transformés en consommateurs et en consommatrices; leur légitimité est réduite de moitié dans une logique de production. Ce glissement, à notre avis, reflète la dichotomie entre les dimensions politiques et technocratiques de la gestion du système sociosanitaire.

the law does refer to the consumer. The distinction is not a trivial one. Whereas " citizen " implies a directing decision-making role, " consumer » is merely a user of service, no more than one-half the buyer-seller equation. In our own political tradition, the label " consumer » carries none of the authority of the citizen. It is my contention that the citizen in health planning has been forced to suffer a role demotion to the status of consumer, and that this role demotion is a central cause of citizen disempowerment (Ellenberger, 1981, p. 50).

Pour Ellenberger (1981), les citoyens et les citoyennes doivent réellement avoir accès au pouvoir. Les priorités des citoyens et des citoyennes diffèrent de celles des technocrates et des producteurs de senvices et leurs intérêts doivent primer sur ceux des autres groupes. Elle a observé que les citoyens et les citoyennes se préoccupent de l'accessibilité des services, du fardeau et de l'impact des soins pour la famille, de la disponibilité de l'information dont ils ont besoin, des délais de traitement, de leur capacité de payer. Pour Ellenberger, les citoyens et les citoyennes doivent affirmer leur souveraineté, former des alliances et des coalitions pour promouvoir et défendre leurs intérêts et s'engager en grand nombre pour participer à la planification des services qui seront alors définis en fonction de leurs véritables besoins. 
Ils doivent également embaucher et diriger les employés qui planifient les services et il leur revient de définir les priorités en fonction « d'objectifs de résultats " sur la santé des personnes, plutôt qu'en fonction des exigences ou des contraintes du système de services (Ellenberger, 1981 , p. 59-60).

\section{CONCLUSION}

À la fin des années 1980, la commission Rochon déplorait des lacunes importantes en matière d'évaluation des politiques, des services et des programmes. Au cours des années 1990, la nécessité d'évaluer les services, les programmes et les politiques afin d'éclairer les choix des décideurs et des décideuses, choix dont ils doivent rendre compte, a été exacerbée par la décroissance des budgets accordés par l'État aux services publics en général et sociosanitaires en particulier. Aujourd'hui, avec l'essor qu'ont connu les pratiques évaluatives, nous sommes en mesure de prendre des décisions éclairées concernant l'avenir des services de santé et des services sociaux. Publication de cadres de référence en évaluation, déploiement de systèmes d'information-clientèle, mise sur pied du Conseil québécois d'agrément, création de l'Institut en santé publique, autant de mesures qui contribuent à mieux connaître la nature, la qualité et l'efficacité des services rendus en fonction des besoins de la population. Mais l'excellence des informations recueillies ne peut suffire à rendre légitimes les décisions qui doivent être prises. Dans une véritable perspective démocratique, le processus de prise de décision doit interpeller l'ensemble des acteurs concernés par les enjeux en cause et engager les citoyens et les citoyennes dans un véritable débat démocratique.

Non seulement les pratiques doivent être révisées, mais les priorités d'intervention deviennent un enjeu de plus en plus important. Quels services, quels programmes doivent être offerts? Quelles sont les approches les plus efficaces? Comment distribuer équitablement les ressources limitées dont nous disposons? L'évaluation des services, des programmes et des politiques doit nous permettre d'engager un véritable débat démocratique, avec les acteurs légitimement concernés, afin de répondre à ces questions.

Alors qu'il semble à première vue plus difficile de rejoindre et d'intéresser la population en général à la planification et à l'évaluation des 
services sociaux et de santé, quelques mesures pourraient permettre d'accroître leur implication et leur engagement dans un véritable débat public et collectif sur l'évaluation, les orientations et les priorités de réorganisation du système sociosanitaire québécois. Une plus grande accessibilité à l'information, un meilleur usage des mécanismes de plaintes, l'utilisation de sondages, d'enquêtes, d'audiences et de consultations publiques, la mise sur pied de comités ou même de commissions d'évaluation des programmes et de révision des politiques, une participation accrue aux conseils d'administration des établissements et des régies régionales sont autant de voies qui peuvent conduire les Québécois et les Québécoises vers un réel débat démocratique sur la qualité et l'avenir de leur réseau de santé et de services sociaux. C'est en multipliant les lieux de négociation et les occasions de débats publics que l'évaluation des politiques, des programmes et des services pourra contribuer à démocratiser les services de santé et les services sociaux.

Mireille TREMBLAY

Directrice générale

Fédération québécoise des CRPDI

\section{Références bibliographiques}

BERGERON, Pierre (1985). « La recherche évaluative au ministère des Affaires sociales ", Carrefour des affaires sociales, vol. 7, n², p. 33-36.

BIBEAU, Gilles (1984). "Le facteur humain en politique : application au domaine de la santé mentale ", Santé mentale au Québec, vol. XI, nº 1 , p. 19-41.

BRASSARD, Lise (1987). La participation : dossier thématique, Québec, Gouvernement du Québec, Commission d'enquête sur les services de santé et les services sociaux.

COMMISSION D'ENQUÊTE SUR LES SERVICES DE SANTÉ ET LES SERVICES SOCIAUX (1987). Résumé du rapport, Gouvernement du Québec.

CONSEIL QUÉBÉCOIS DE LA RECHERCHE SOCIALE (1995). Évaluer : pourquoi? Actes du colloque tenu à Chicoutimi le 23 mai 1995, Gouvernement du Québec. 
Corin, Ellen, Gilles BIBEAU, Jean-Claude MARTIN et Robert LAPLANTE (1990). Comprendre pour soigner autrement, Montréal, Presses de l'Université de Montréal.

D'ANNUNZIO, Suzanne (1986). L'expérience en évaluation de programme au ministère de la Santé et des Services sociaux, communication présentée à la Conférence annuelle de la Société canadienne d'évaluation de programme, avril 1986, Québec, Direction de l'évaluation et de la planification, ministère de la Santé et des Services sociaux.

Demers, L. (1987). L'évaluation, Gouvernement du Québec, Commission d'enquête sur les services de santé et les services sociaux.

DESLAURIERS, J.-P. (1987). Les méthodes de la recherche qualitative, Sillery, Presses de l'Université du Québec.

ELLENBERGER, Dorothy (1981). "Participation is not enough", dans B. Checkoway, Citizens and Health Care, Participation for Social Change, Toronto, Pergamon, p. 49-61.

FONDS DE LA RECHERCHE EN SANTÉ DU QUÉBEC (1990). Profil de la recherche en santé au Québec, Montréal, Fonds de la recherche en santé au Québec.

FRENCH, Richard D. (1987). Le ministre et l'analyse des politiques publiques, allocution prononcée devant les membres de l'Institut d'administration publique du Canada, avril 1987, Gouvernement du Québec, Office des ressources humaines.

GARnEAU, Hélène, André PoItras et Louis SAVARD (1986). L'évaluation de programmes : son implantation dans trois ministères - Justice, Santé et Services sociaux, Sécurité du revenu, Gouvernement du Québec, Conseil du trésor.

GAUTHIER, Benoît (1982). Méta-évaluation en affaires sociales : analyse de cent cas de recherches évaluatives, analyse réalisée pour le compte du Secrétariat à la coordination de la recherche et du Conseil québécois de la recherche sociale, Hull.

GodBout, Jacques, Murielle Leduc et Jean-Pierre Collin (1987). La face cachée du système, rapport présenté à la Commission d'enquête sur la santé et les services sociaux, Gouvernement du Québec.

Godue, Charles et Laurent Chambaud (1981). " Les risques d'une politique de santé ", Revue canadienne de politique sociale, no 18, p. 112-121.

GUBA, E. et Y. LINCOLN (1989). Fourth Generation Evaluation, Newbury Park, CA, Sage.

LAFLAMME, Michel (1987). Support des méthodes qulitatives dans l'évaluation de programme, Québec, CSSQ. 
LAMOUREUX, Jocelyne (1994). Le partenariat à l'épreuve, Montréal, Éditions Saint-Martin.

LAPLANTE, Laurent (1987). L'évaluation des programmes publics au Québec, où en sommes-nous?, Actes du colloque, Québec, Gouvernement du Québec, Conseil du trésor, Centre pour le développement de la productivité dans le secteur public.

LESEMANN, Frédéric et Jocelyne LAMOUREUX (1987). Le rôle et le devenir de l'État-Providence, rapport remis à la Commission d'enquête sur la santé et les services sociaux, Québec, Gouvernement du Québec.

Ministère de La Santé et des Services sociaux (1992). Politique de la santé et du bien-être, Gouvernement du Québec.

MINISTÈRE DE LA SANTÉ ET DES SERVICES SOCIAUX (1990). Une réforme axée sur le citoyen, Gouvernement du Québec.

MINISTÈre de LA SANTÉ ET DES SeRVICES SOCIAUX (1989). Orientations en matière de santé et de services sociaux, Gouvernement du Québec.

NADEAU, Marc-André (1988). L'évaluation de programmes : théorie et pratique, Sainte-Foy, Presses de l'Université Laval.

ORGANISATION MONDIALE DE LA SANTÉ (1987). Évaluation de la santé pour tous d'ici l'an 2000, septième rapport sur la situation sanitaire dans le monde, vol. 1, Analyse mondiale, Genève, OMS.

ORGANISATION MONDIALE DE LA SANTÉ (1981). L'évaluation des programmes de santé : principes directeurs pour son application dans le processus gestionnaire pour le développement sanitaire national, Genève.

OUELLET, Francine et Christiane LAMPRON (1987). Bilan des évaluations portant sur les services sociaux, rapport présenté à la Commission d'enquête sur la santé et les services sociaux, Gouvernement du Québec.

PATTON, Michael (1987). Creative Evaluation, Beverley Hills, CA, Sage.

PATTON, Michael (1987). How to use qualitative methods in evaluation, Beverley Hills, CA, Sage.

PINEAULT, Raynald et Carole DAVELUy (1986). La planification de la santé, Montréal, Agence d'Arc.

RENAUD, Gilbert (1984). À l'ombre du rationalisme, Montréal, Éditions SaintMartin.

SOULET, M.-H. (1987). " La recherche qualitative ou la fin des certitudes ", dans J.-P. Deslauriers, Les méthodes de la recherche qualitative, Sillery, Presses de l'Université du Québec.

VON SCHOENBERG, Brigitte (1985). Les points de vue des clients et des citoyens : leur place dans l'évaluation des programmes, Québec, MAS, Service de l'évaluation des programmes de services sociaux. 